\title{
Revista Colombiana de

\section{Evaluación y seguimiento de pacientes ambulatorios con diabetes mellitus tipo 2 mediante control metabólico individualizado y variables antropométricas}

\author{
Lina P. León-Sierra ${ }^{\mathrm{a}, *}$, Carolina Jiménez-Rodríguez ${ }^{\mathrm{a}}$, Jorge J. Coronado-Tovar ${ }^{\mathrm{b}}$, \\ Nelcy Rodríguez-Malagón ${ }^{\mathrm{c}}$ y Análida E. Pinilla-Roa ${ }^{\mathrm{a}}$
}

\author{
a Departamento de Medicina Interna. Facultad de Medicina. Universidad Nacional de Colombia. Bogotá, Colombia \\ b Facultad de Medicina. Universidad Nacional de Colombia. Bogotá, Colombia \\ c Departamento de Estadística. Facultad de Ciencias. Universidad Nacional de Colombia. Bogotá, Colombia
}

Recibido el 30 de junio de 2017; aceptado el 3 de diciembre de 2018

Disponible en Internet el 23 de abril de 2019

\author{
PALABRAS CLAVE \\ Atención \\ ambulatoria; \\ Diabetes mellitus tipo \\ 2; \\ Hemoglobina A \\ glicosilada; \\ Educación del \\ paciente
}

\begin{abstract}
Resumen
Objetivo: evaluar el perfil metabólico y antropométrico, por metas individualizadas, en pacientes con diabetes mellitus tipo 2.

Métodos: estudio observacional prospectivo de pacientes con diabetes mellitus tipo 2 que asistieron a consulta especializada e ingresaron desde junio 2015 hasta julio 2016 a un seguimiento durante seis meses, categorizados según guías clínicas: 1 . Adulto saludable, 2 . Adulto complejo y adulto mayor saludable, 3. Adulto mayor complejo y 4 . Adulto mayor muy complejo. Durante los controles recibieron educación y se registraron: peso, índice de masa corporal, perímetro abdominal, porcentaje de grasa, glucemia, hemoglobina A glicosilada, colesterol, LDL, HDL y triglicéridos. Se compararon metas individualizadas entre categorías.

Resultados: se analizaron 36 pacientes. La comorbilidad y complicación más frecuentes fueron la hipertensión arterial $(63,9 \%)$ y la neuropatía diabética $(64.7 \%)$, respectivamente.

Al sexto mes, la meta de hemoglobina A glicosilada se logró en $58,3 \%$; se observó reducción de peso $1 \mathrm{~kg}$ y de índice de masa corporal $0,5 \mathrm{~kg} / \mathrm{m}^{2}$, con promedio $27,6 \mathrm{~kg} / \mathrm{m}^{2}$; el porcentaje de grasa abdominal y el perímetro abdominal se mantuvieron estables. Por metas individualizadas la categoría 1 tuvo mayor cumplimiento de meta en glucemia basal y la categoría 3 en hemoglobina A glicosilada.

Conclusiones: el seguimiento y la educación permanente a cada paciente y familia permiten valorar la mejoría de metas metabólicas y variables antropométricas, facilitan el fortalecimiento de la relación médico-paciente y permiten evaluar el impacto de las intervenciones.
\end{abstract}

\footnotetext{
* Autor para correspondencia.

Correo electrónico: linapaola.leon@gmail.com (L.P. León-Sierra).
} 


\section{KEY WORDS}

Out-patient Care; Diabetes mellitus type 2; Glycosylated haemoglobin A; Patient education

Sin embargo, es necesario organizar programas interdisciplinarios para optimizar el control de pacientes con diabetes mellitus tipo 2 .

(c) 2019 Sociedad Colombiana de Cardiología y Cirugía Cardiovascular. Publicado por Elsevier España, S.L.U. Este es un artículo Open Access bajo la licencia CC BY-NC-ND (http:// creativecommons.org/licenses/by-nc-nd/4.0/).

Evaluation and follow-up of ambulatory patients with diabetes mellitus type 2 by means of individualised metabolic control and anthropometric measurements

\begin{abstract}
Objective: To evaluate the metabolic and anthropometric profile, by individualised targets, in patients with diabetes mellitus type 2 .

Methods: An observational, prospective study carried out on patients with diabetes mellitus type 2 that attended a specialised clinic and were enrolled from June 2015 to July 2016, to a follow-up of six months They were classified according to clinical guidelines as; 1 . healthy adult, 2. Complex adult and older health adult, 3. Complex older adult, and 4. Very complex older adult. During the follow-up they received education and a record was made of; weight, body mass index, abdominal circumference, percentage fat, blood glucose, glycosylated haemoglobin A, cholesterol, LDL, HDL, and triglycerides. The individualised targets were compared between categories.

Results: The analysis included a total of 36 patients. The most frequent comorbidity and complication was arterial hypertension $(63.9 \%)$, and diabetic neuropathy $(64.7 \%)$, respectively.

At the sixth month, the glycosylated haemoglobin A target was achieved in $58.3 \%$. A weight reduction of $1 \mathrm{~kg}$ was observed, as well as a reduction of $0.5 \mathrm{~kg} / \mathrm{m}^{2}$ in $B M I$, with a mean of $27.6 \mathrm{~kg} / \mathrm{m}^{2}$, The percentage of abdominal fat and the abdominal circumference remained stable. By individualised targets, Category 1 had a greater compliance in baseline blood glucose, and Category 3 in glycosylated haemoglobin A.

Conclusions: The follow-up and education of each patient and family help to improve the metabolic targets and the anthropometric variables. This also strengthened the doctor-patient relationship, and enabled the impact of the interventions to be evaluated. However, interdisciplinary program need to be organised in order to optimise control in patients with diabetes mellitus type 2 .

(c) 2019 Sociedad Colombiana de Cardiología y Cirugía Cardiovascular. Published by Elsevier España, S.L.U. This is an open access article under the CC BY-NC-ND license (http:// creativecommons.org/licenses/by-nc-nd/4.0/).
\end{abstract}

\section{Introducción}

La enfermedad cardiovascular es la principal causa de muerte y discapacidad en las personas con diabetes mellitus tipo 2, además, esta es más prevalente cuando la persona presenta esta dicha enfermedad que cuando no ${ }^{1,2}$. Por lo anterior, se hace necesario el desarrollo de consultas especializadas o, mejor aún, programas para la prevención primaria y secundaria, detección temprana y control de los factores de riesgo modificables, para disminuir o retardar la progresión de complicaciones microvasculares y macrovasculares, que no solo generan alto costo sino impacto social, familiar y laboral; así, el paciente requiere seguimiento periódico por un equipo que gestione su salud y lo eduque para apropiar estrategias de autocuidado ${ }^{3-5}$.

Estos programas deben ser desarrollados por grupos interdisciplinarios versus multidisciplinarios que articulan la acción profesional de enfermeros, médicos generales, internistas, endocrinólogos, cardiólogos, nutricionistas, psicólogos, entre otros, de tal forma que se garantice el control y seguimiento mediante evaluaciones periódicas, para instaurar las intervenciones farmacológicas y no farmacológicas de forma individualizada, acorde con los problemas de cada paciente según su contexto (cultural, social, económico, nivel de escolaridad) e historia clínica. Por esto se requiere un abordaje multidimensional no realizable desde cada disciplina o profesión aisladas, sino a partir de la cooperación y construcción mutuas ${ }^{6,7}$. La evidencia actual demuestra que para alcanzar las metas del tratamiento de un paciente ambulatorio, con diabetes mellitus tipo 2, es necesario que cada paciente y su familia se apropien de temas como: autocuidado, automonitorización, técnica de aplicación de insulina, corrección de hiperglucemia o hipoglucemia y hábitos de un estilo de vida saludable para mejorar los desenlaces clínicos asociados al control permanente de metas e impactar en su calidad de vida ${ }^{8-10}$.

Para conocer el control metabólico de los pacientes con diabetes mellitus tipo 2 , se llevó a cabo este estudio observacional prospectivo de pacientes, en una consulta especializada en esta patología, de Medicina interna, en un hospital de tercer nivel de atención, sin contar con equipo de otros profesionales como psicología, nutrición, terapia 
física, entre otros; los resultados permitirían mejorar la planificación y la programación de la atención en salud de pacientes ambulatorios, con diabetes mellitus tipo 2 , en diferentes niveles de atención y en una consulta especializada, con el fin de impactar en el control metabólico de la enfermedad y las variables antropométricas (peso, índice de masa corporal, porcentaje de grasa abdominal, perímetro de la cintura). Se buscó demostrar la importancia del seguimiento regular de cada paciente para brindar educación junto a variables metabólicas y antropométricas como parte de un tratamiento integral efectivo.

\section{Métodos}

Estudio observacional prospectivo de pacientes, del régimen subsidiado, que ingresaron a la consulta de diabetes mellitus en Medicina Interna del Hospital El Tunal en Bogotá D.c., Colombia, desde junio de 2015 a julio de 2016; se sistematizó el seguimiento individual durante seis meses (mes 1, 3 y 6). La recolección de datos del último paciente fue hasta diciembre de 2016. Para garantizar el seguimiento regular se organizó la programación de los controles mediante la asignación de la cita al culminar cada consulta. Participaron pacientes mayores de 18 años, con diagnóstico de diabetes mellitus tipo 2 que asistían a esta consulta especializada, según los criterios establecidos por la Asociación Americana de Diabetes $(2016)^{8}$, que tuvieran al menos tres meses de tratamiento para diabetes mellitus tipo 2 y que firmaron un consentimiento informado. Se excluyeron pacientes con diabetes mellitus tipo 1 , gestantes, enfermedad renal crónica estadio $\mathrm{V}$, amputación transfemoral unilateral o bilateral y síndrome nefrótico.

Se construyó y aplicó un instrumento con datos de identificación del paciente, variables sociodemográficas y antropométricas: peso, índice de masa corporal (IMC), porcentaje de grasa abdominal y perímetro abdominal (normal en hombres $<94 \mathrm{~cm}$ y en mujeres $<90 \mathrm{~cm})^{10}$. El IMC $\left(\mathrm{kg} / \mathrm{m}^{2}\right)$ se dividió en 3 grupos según fuese normal (IMC menor de 25), preobesidad o sobrepeso (IMC entre 25 y 29,9) u obesidad (IMC mayor de 30); además, en el examen físico se registraron: presión arterial, pulsos de miembros inferiores, comorbilidades (hipertensión arterial, falla cardiaca, evento cerebrovascular, enfermedad arterial periférica, alteración tiroidea, retinopatía diabética, hiperlipidemia, enfermedad renal crónica); tratamiento farmacológico para la diabetes mellitus tipo 2 (antidiabéticos orales: metformina, inhibidores de dipeptidildipeptidasa IV, análogos de GLP1, insulinas análogas de corta y larga acción; a los pacientes que venían de otra consulta con glibenclamida se les retiró); resultados de laboratorio del control metabólico -glucemia basal, hemoglobina A glicosilada y perfil lipídico.

El instrumento de cada paciente se diligenció al ingreso, el tercer y el sexto mes. El grupo de investigación integró y adaptó las recomendaciones de diversas guías clínicas; por consiguiente, se analizó la historia clínica de cada paciente para definir a qué categoría correspondía con sus respectivas metas de glucemia basal y hemoglobina A glicosilada para el control, seguimiento y análisis de los datos (edad, comorbilidades, complicaciones, déficit cognitivo y red de apoyo, entre otras (tabla 2$)^{8-13}$.
Tabla 1 Características sociodemográficas de los pacientes

\begin{tabular}{lll}
\hline & No. & $\%$ \\
\hline $\begin{array}{l}\text { Género } \\
\quad \text { Mujeres }\end{array}$ & 22 & \\
$\quad$ Hombres & 14 & 61,1 \\
Edad & & 38,9 \\
$\quad<65$ años & 19 & 52,8 \\
$\quad>65$ años & 17 & 47,2 \\
Estrato socioeconómico & & \\
1 & 16 & 44,4 \\
2 & 16 & 44,4 \\
3 & 4 & 11,1 \\
Nivel de formación & & 13,9 \\
$\quad$ Ninguno & 5 & 63,9 \\
Primaria & 23 & 19,4 \\
Secundaria & 7 & 2,8 \\
Técnico & 1 & 0 \\
Universitario & 0 & \\
Tiempo de diagnóstico & & 52,8 \\
$\quad<10$ años & 19 & 47,2 \\
$\quad>10$ años & 17 & \\
\hline & &
\end{tabular}

Todos los exámenes se procesaron en el laboratorio del hospital. La glucemia fue medida por método enzimático con uso de hexoquinasa y los lípidos (colesterol total, HDL, triglicéridos) por método enzimático colorimétrico. Se calculó el LDL mediante fórmula de Friedewald ${ }^{14}$ y la hemoglobina A glicosilada por inmunoensayo por turbidimetría de inhibición, todos medidos en el equipo Cobas-c 501.

Para la confirmación de comorbilidades y complicaciones por diabetes mellitus tipo 2 se estudiaron la falla cardiaca por síntomas según los Criterios de Framingham ${ }^{15}$ y la función ventricular por ecocardiografía (y electrocardiografía); la presión arterial tomada con Tensiómetro WelchAllyn Ds4411 calibrado y la enfermedad tiroidea por clínica y medición de TSH y T4 libre. La enfermedad arterial periférica se valoró por interrogatorio de claudicación intermitente. En cuanto a la palpación de pulsos de extremidades inferiores (femorales, poplíteos, pedios y tibiales posteriores) si hubo alteración se confirmó mediante Doppler arterial de miembros inferiores ${ }^{16}$; la retinopatía fue diagnosticada por un oftalmólogo ${ }^{17}$; la nefropatía diabética con tasa de filtración glomerular por CKD-EPI menor a $60 \mathrm{ml} / \mathrm{min} / 1,72 \mathrm{~m}^{2}$ y la medición de albuminuria/creatinuria ${ }^{18}$; la neuropatía por la presencia de disestesias estudiada con prueba de monofilamento de Semmes-Weinstein 5.07 de $10 \mathrm{~g}$ y diapasón de $128 \mathrm{~Hz}$; el diagnóstico se confirmó si alguna estaba alterada ${ }^{19}$. Para las medidas antropométricas se utilizó una báscula marca TANITAS Fit Scan Body Fat Monitor BF-679F para peso y porcentaje de grasa abdominal y una cinta métrica de fibra de vidrio para el perímetro de la cintura (en el punto medio entre la última costilla y la parte superior de la cresta ilíaca) ${ }^{20}$.

Durante el seguimiento se brindó educación a cada paciente y su familia sobre la automonitorización si recibía insulina, técnica de aplicación de la misma, prevención del pie diabético y hábitos de vida saludable; además, se hizo acompañamiento telefónico para aclarar inquietudes y confirmar la asistencia a los controles. 
Tabla 2 Categorías de metas individualizadas para hemoglobina glicosilada, glucemia y glucometría

\begin{tabular}{|c|c|c|c|}
\hline \multirow[t]{2}{*}{ Categoría } & \multirow{2}{*}{$\begin{array}{l}\text { Metas } \\
\text { hemoglobina } \\
\text { glicosilada }\end{array}$} & \multirow[b]{2}{*}{ Glucemia basal } & \multirow[b]{2}{*}{$\begin{array}{l}\text { Glucometría basal } \\
\text { o preprandial }\end{array}$} \\
\hline & & & \\
\hline 1. Adulto saludable $^{2}$ & $6,5-7 \%$ & $70-110 \mathrm{mg} / \mathrm{dl}$ & $80-120 \mathrm{mg} / \mathrm{dl}$ \\
\hline $\begin{array}{l}\text { 2. Adulto complejo (con lesión de órgano } \\
\text { blanco/comorbilidades) y } \\
\text { Adulto mayor saludable (> } 65 \text { años) }\end{array}$ & $7,1-7,5 \%$ & $90-130 \mathrm{mg} / \mathrm{dl}$ & $100-140 \mathrm{mg} / \mathrm{dl}$ \\
\hline $\begin{array}{l}\text { 3. Adulto mayor complejo (con lesión de } \\
\text { órgano blanco/comorbilidades) }\end{array}$ & $7,5-8 \%$ & $90-150 \mathrm{mg} / \mathrm{dl}$ & $100-160 \mathrm{mg} / \mathrm{dl}$ \\
\hline $\begin{array}{l}\text { 4. Adulto mayor muy complejo (con lesión de } \\
\text { órgano blanco/comorbilidades en etapas } \\
\text { finales, deterioro cognitivo moderado a } \\
\text { severo, dependencia funcional) }\end{array}$ & $8,1-8,5 \%$ & $100-180 \mathrm{mg} / \mathrm{dl}$ & $110-190 \mathrm{mg} / \mathrm{dl}$ \\
\hline
\end{tabular}

Se construyó y analizó la base de datos en el Programa Microsoft Excel versión 2016; para el análisis descriptivo de las variables cuantitativas discretas o continuas se utilizaron medidas de tendencia central y dispersión. Las variables categóricas fueron descritas mediante proporciones.

El protocolo de investigación fue aprobado por el Comité de Ética de la Facultad de Medicina y el Comité de Investigación del Hospital El Tunal. Se consideraron las "Normas Científicas, Técnicas y Administrativas para la Investigación en Salud' ' establecidas en la Resolución 008430 de 1993 del Ministerio de Salud Colombiano.

\section{Resultados}

Ingresaron 52 pacientes que cumplían criterios de inclusión y se excluyeron 14 pacientes: 11 pérdidas de seguimiento (cambio de Empresas Prestadoras de Salud -EPS- o inasistencia a controles), 1 por falta de adherencia farmacológica, 1 por múltiples hospitalizaciones en adulto mayor muy complejo (tabla 2) y, otro, por pobre red de apoyo; predominaron las mujeres $(61,1 \%)$, estratos socioeconómicos 1 y $2(44,4 \%)$, escolaridad primaria $(63,9 \%)$ y tiempo de diagnóstico menor a 10 años (52,8\%); además, menores de 65 años $(52,8 \%)$, con edad promedio 63 años (tabla 1).

Las comorbilidades fueron: hipertensión arterial $(63,9 \%)$, hipertrigliceridemia (50\%), hipotiroidismo $(38,9 \%)$, hipercolesterolemia $(33,3 \%)$ y falla cardiaca $(8,3 \%)$. Las complicaciones más frecuentes: neuropatía periférica $64,7 \%$ por la prueba del diapasón de $128 \mathrm{~Hz}$ de los cuales $11,7 \%$ tenían alteración del monofilamento de Semmes-Weinstein 5.07 de $10 \mathrm{~g}$; retinopatía $(16,7 \%)$; nefropatía $(14,7 \%)$; enfermedad coronaria $(8,3 \%)$ y ningún caso de enfermedad arterial periférica.

\section{Variables antropométricas}

Se encontró sobrepeso (preobesidad) ${ }^{20}$ en $38,9 \%$ y obesidad en $41,7 \%$ con predominio en mujeres; porcentaje de grasa abdominal en rango saludable $36,4 \%$ (5 mujeres y 7 hombres); 33,3\% en obesidad (10 mujeres, 1 hombre) y perímetro abdominal elevado $72,7 \%$ de mujeres y en $42,9 \%$ de hombres. A seis meses, se observó una reducción de 1 kg y de $0,5 \mathrm{~kg} / \mathrm{m}^{2}$ en índice de masa corporal con promedio $27,6 \mathrm{~kg} / \mathrm{m}^{2}$; el porcentaje de grasa y perímetro abdominal se mantuvieron estables (tabla 3 ).

\section{Control metabólico}

El promedio de la glucemia basal al ingreso fue $144,7 \mathrm{mg} / \mathrm{dl}$ y hemoglobina A glicosilada $8,4 \%$, con disminución de $0,6 \%$ a los seis meses. Al ingreso las mujeres presentaron hemoglobina A glicosilada de $8,5 \%$ y los hombres de $8,1 \%$, con reducción de $0,9 \%$ y $0,2 \%$, respectivamente (fig. 1 ).

En el seguimiento general de los pacientes, con relación al porcentaje de cumplimiento de metas, se encontró que al final del sexto mes $58,3 \%$ de la muestra había cumplido las metas de hemoglobina A glicosilada, logrando un aumento del $16,7 \%$ respecto al ingreso.

El seguimiento del perfil lipídico se completó en $88,9 \%$ de los pacientes. Se observó aumento del $14,7 \mathrm{mg} / \mathrm{dl}$ en colesterol total; $8,1 \mathrm{mg} / \mathrm{dl}$ en triglicéridos; $14,3 \mathrm{mg} / \mathrm{dl}$ en LDL y reducción del $1,4 \mathrm{mg} / \mathrm{dl}$ en el $\mathrm{HDL}$; lo anterior puede correlacionarse con la dificultad para el seguimiento estricto de la adherencia a las estatinas en este grupo de pacientes estudiado.

Con base en las categorías descritas (tabla 2$)^{8}$, Los pacientes se distribuyeron así: 16 en categoría $1 ; 6$ en categoría 2, 14 en categoría 3 y ninguno en categoría 4.

En la categoría 1, al ingreso, 5 pacientes se encontraban en metas de hemoglobina A glicosilada $(31,3 \%)$ y a los 6 meses 10 (62,5\%).

En la categoría 2, al ingreso el $50 \%$ se encontraba en metas de glucemia basal y hemoglobina A glicosilada, sin cambios a los 6 meses.

En la categoría 3 , al ingreso 8 pacientes $(57,1 \%)$ se encontraban en meta de glucemia basal y 7 (50\%) en hemoglobina A glicosilada; a los 6 meses, hubo un aumento en el cumplimiento de metas de glucemia basal en $14,3 \%$ y en hemoglobina A glicosilada de 7,1\% (fig. 2). 
Tabla 3 Seguimiento de las variables antropométricas

\begin{tabular}{|c|c|c|c|c|c|c|c|}
\hline \multirow[b]{2}{*}{ Variable } & \multicolumn{2}{|c|}{ Ingreso } & \multicolumn{2}{|c|}{ Tercer mes } & \multicolumn{2}{|c|}{ Sexto mes } & \multirow{2}{*}{$\begin{array}{l}\text { Diferencia } \\
\text { ingreso-sexto mes }\end{array}$} \\
\hline & $X^{*}$ & $M e^{* *}$ & $X^{*}$ & $M e^{* *}$ & $X^{*}$ & $M e^{* *}$ & \\
\hline Peso $(k g)$ & 71,5 & 69,1 & 71,3 & 68,4 & 70,5 & 67,0 & $-1,0 \mathrm{Kg}$ \\
\hline $\begin{array}{l}\text { Índice de masa corporal }\left(\mathrm{kg} / \mathrm{m}^{2}\right) \\
\% \text { grasa abdominal }\end{array}$ & 29,6 & 28,1 & 29,4 & 27,6 & 29,1 & 27,6 & $-0,5 \mathrm{Kg} / \mathrm{m}^{2}$ \\
\hline Hombres $(n=13)$ & 24,2 & 24,2 & 23,6 & 22,9 & 23,7 & 24,3 & $-0,5 \%$ \\
\hline Mujeres $(n=20)$ & 37,8 & 40,1 & 38,3 & 41,8 & 38,0 & 40,0 & $+0,2 \%$ \\
\hline Perímetro abdominal (cm) & & & & & & & \\
\hline Hombres $(n=13)$ & 95,7 & 94 & 97,0 & 96 & 95,8 & 94 & $+0,1 \mathrm{~cm}$ \\
\hline Mujeres $(n=21)$ & 101,5 & 99 & 102,1 & 102 & 101,0 & 101 & $-0,5 \mathrm{~cm}$ \\
\hline
\end{tabular}

* $X$ : media ** Me: mediana

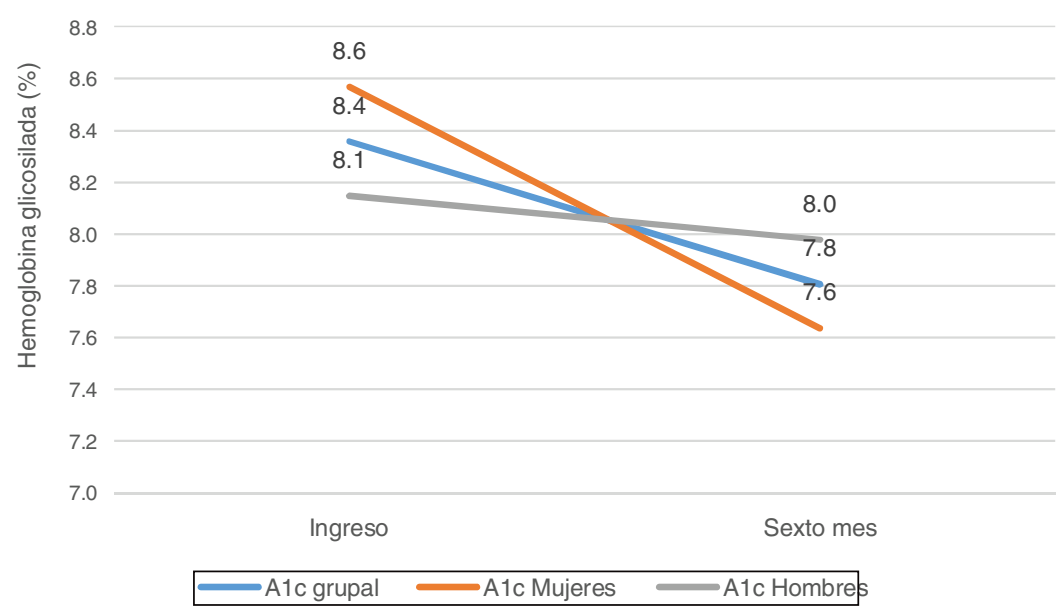

Figura 1 Seguimiento de hemoglobina glicosilada grupal y por género.

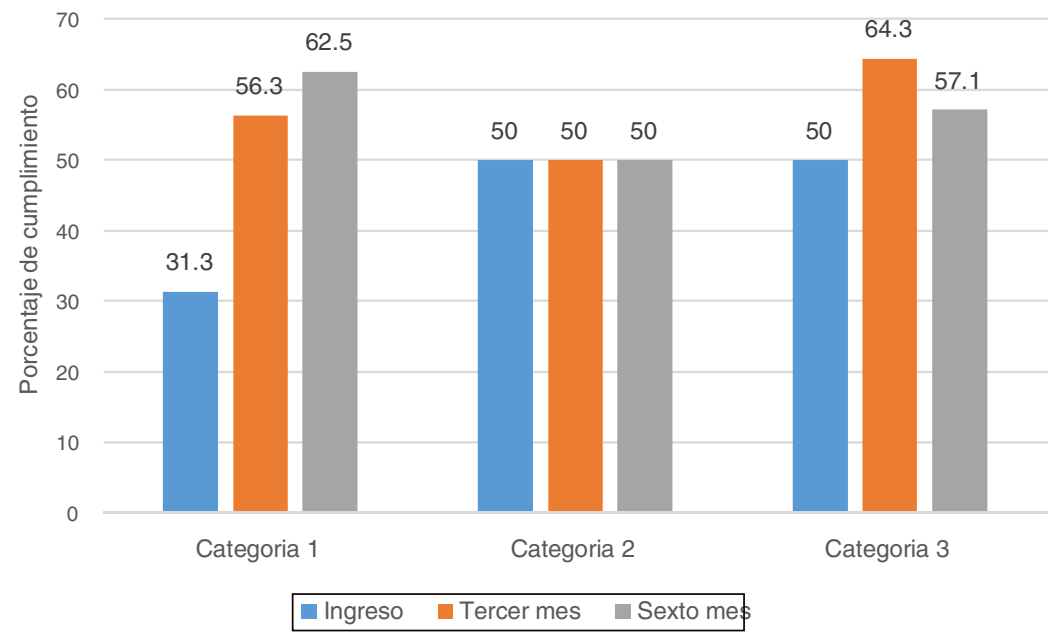

Figura 2 Cumplimiento de metas individualizadas de hemoglobina A glicosilada.

\section{Discusión}

La diabetes mellitus tipo 2 es una enfermedad sistémica con alta morbimortalidad; su descontrol se relaciona con complicaciones microvasculares y macrovasculares e incrementa el número de hospitalizaciones con estancia prolongada ${ }^{21}$ y carga económica al sistema de salud ${ }^{22}$.
Este estudio prospectivo de pacientes ambulatorios con diabetes mellitus tipo 2 , con educación continua del paciente y su familia, seguidos en una consulta especializada de Medicina interna mostró que el equipo médico de la consulta, conformado por internista/docente, residentes de medicina interna y estudiante de internado es insuficiente para alcanzar las metas de control metabólico y 
antropométrico; de ahí la importancia del trabajo y seguimiento interdisciplinario para alcanzar las metas individualizadas del control metabólico, reducir las complicaciones, mejorar la calidad de vida relacionada con la salud y reducir costos al sistema ${ }^{8,22}$.

A pesar de la amplia evidencia que fundamenta las guías de práctica clínica para el tratamiento y seguimiento individualizado, la proporción de pacientes que continúan fuera de metas es alta ${ }^{23-25}$. Estudios transversales retrospectivos en Latinoamérica y España reportaron que del 31,8\% al $60,7 \%$ estaban en metas para hemoglobina A glicada ${ }^{22,26}$. Aun así, los programas especializados siguen demostrando un impacto positivo sobre el control metabólico ${ }^{27}$; investigaciones con seguimiento intensivo interdisciplinario reportaron una reducción de hemoglobina $A$ glicosilada de $2,3 \%$ a seis meses y $2,5 \%$ a 12 meses $^{28,29}$.

En Colombia, estudios en pacientes hospitalizados como el de Alba et al. (2009) ${ }^{21}$ y Osuna et al. (2014) ${ }^{30}$, tomaron como meta del control de hemoglobina A glicosilada un solo punto de corte $\leq 7 \%$ para todo perfil de paciente; sin embargo, diversas guías clínicas proponen categorizar según el perfil del paciente (años de diagnóstico de la DM2, comorbilidades, lesiones microvasculares y macrovasculares, trastorno cognitivo, expectativa de vida, entre otras $^{8,10-13}$.

Se sabe que por cada $1 \%$ de reducción de hemoglobina A glicosilada, disminuye la progresión de complicaciones microvasculares en $37 \%$ y de mortalidad en $21 \%^{31,32}$. Pese a que aún no se haya demostrado el impacto del control glucémico estricto sobre las lesiones macrovasculares ${ }^{33,34}$, el nivel de hemoglobina A glicosilada se asocia con amputaciones, muerte por enfermedad vascular periférica e infarto agudo de miocardio fatal y no fatal, lo cual lo convierte en un factor de riesgo cardiovascular independiente ${ }^{35}$; esta es meta fundamental, así como la presión arterial, los lípidos y al abandono del tabaquismo ${ }^{36}$.

Por lo anterior, se realizó una construcción propia con cuatro categorías de pacientes (tabla 2$)^{8,10-13}$ en la que, además, se integraron las metas individualizadas del perfil lipídico, en dos categorías según la presencia de enfermedad cardiovascular definida como infarto agudo de miocardio, enfermedad arterial periférica y accidente cerebrovascular: LDL en diabetes mellitus tipo $2<100 \mathrm{mg} / \mathrm{dl}$; en diabetes mellitus tipo 2 con enfermedad cardiovascular $<70 \mathrm{mg} / \mathrm{dl}$; colesterol no $\mathrm{HDL}<130 \mathrm{mg} / \mathrm{dl}$ en diabetes mellitus tipo 2 y en diabetes mellitus tipo 2 con enfermedad cardiovascular $<100 \mathrm{mg} / \mathrm{dl}$; HDL $>50 \mathrm{mg} / \mathrm{dl}$ mujeres y $>40$ hombres y triglicéridos $<150 \mathrm{mg} / \mathrm{dl}$ para los dos grupos, los cambios en lípidos no fueron significativos, por lo que no se contemplaron para el análisis estadístico ${ }^{8,37}$.

Se incluyeron las variables antropométricas (peso, índice de masa corporal, porcentaje de grasa abdominal y perímetro abdominal) relacionados con pobre control metabólico; un índice de masa corporal elevado se asocia con mayor riesgo de enfermedad cardiovascular (HR: 1,34-2,45), enfermedad cerebrovascular (HR: 1,30-2,00), lesión renal (HR: $1,31-2,23)$ y pie diabético (HR: $0,41-2,95)^{38}$. Asimismo, el perímetro abdominal y el porcentaje de grasa abdominal son variables superiores para predecir la aparición de patologías cardiometabólicas ${ }^{39-41}$ y mortalidad relacionada con enfermedad cardiovascular en poblaciones sanas ${ }^{42,43}$. La medición del perímetro abdominal es compleja y tiene alta variabilidad interobservador, más aún en pacientes obesos dada la ubicación difícil de los reparos anatómicos y la técnica adecuada $^{20,44}$. La medición del porcentaje de grasa abdominal por bioimpedanciometría es una medida más objetiva que el perímetro abdominal ${ }^{45}$.

De otra parte, la educación al paciente también es un pilar fundamental en los programas especializados en diabetes mellitus tipo 2 para lograr las metas, específicamente reducir la hemoglobina $\mathrm{A}$ glicosilada ${ }^{46}$, por lo que los sistemas de salud e instituciones deben apoyar la gestión estructurada de la estratificación del riesgo, la atención y el servicio al paciente, así como, la organización de equipos ínter- y transdisciplinarios ${ }^{47}$. Este estudio corroboró la necesidad de estos equipos para optimizar el tiempo, la consecución y la estabilidad de las metas individualizadas ${ }^{4,8,10-13}$.

El seguimiento a seis meses permitió valorar la mejoría de la hemoglobina $\mathrm{A}$ glicosilada, con un incremento en el número de pacientes que lograron las metas, principalmente en las categorías 1 y 3 , y la reducción global del peso. Por consiguiente, se propone el control y seguimiento organizado de los pacientes con diabetes mellitus tipo 2, enfocado en la prevención primaria y secundaria más que terciaria, con énfasis en educación continuada del paciente y su familia en automonitorización, técnica de aplicación de insulina, prevención y reconocimiento de los síntomas de hipoglucemia y su tratamiento inmediato, autocuidado de pies y adherencia farmacológica, entre otros ${ }^{48}$.

En cada visita es necesario evaluar las variables antropométricas descritas, realizar un examen físico completo buscando, por ejemplo, lesión de órgano blanco, lipohipertrofia o pie diabético con pruebas como vibración o monofilamento. En el seguimiento metabólico se debe verificar la tabla de automonitorización y solicitar al ingreso y de forma trimestral glucemia basal, hemoglobina $A$ glicosilada, perfil lipídico y función renal con albuminuria/creatinuria. La evaluación de los factores de riesgo cardiovascular orientará la necesidad de estudios especializados. Todos los pacientes deben ser valorados por Nutrición y Oftalmología, y además recibir orientación sobre vacunas y actividad física. Si el paciente alcanza las metas individualizadas el médico podrá espaciar los controles, por ejemplo, hemoglobina A glicosilada cada seis meses $^{5,8,10,11,49}$.

Finalmente, se insiste en que las metas del tratamiento deben ser establecidas de forma individualizada de acuerdo a edad, presencia de comorbilidades, complicaciones, expectativa de vida y apoyo social; estas metas han de ser explicadas y apropiadas por el paciente y su familia. Con base en estas decisiones, se realizarán de forma oportuna ajustes farmacológicos necesarios hasta lograr las metas definidas y trabajar para mantenerlas.

Se invita a la comunidad académica a planear estudios prospectivos de seguimiento de pacientes con diabetes mellitus tipo 2 a mediano y largo plazo para evaluar y definir el impacto de las intervenciones, en diversas poblaciones de pacientes. Asimismo, se recomienda recuperar la relación médico/paciente/familia mediante la continuidad del seguimiento con el médico tratante, en particular, con un internista como especialista integrador de los problemas del 
paciente adulto y de la acción de diversos especialistas y subespecialistas ${ }^{50}$.

\section{Conclusiones}

Este es el primer estudio de seguimiento prospectivo en pacientes ambulatorios con diabetes mellitus tipo 2 en Colombia; la mayoría de estudios que se encuentran en la literatura son transversales o retrospectivos.

El seguimiento del tratamiento farmacológico y no farmacológico y la educación permanente de cada paciente y su familia permitió valorar la mejoría de la hemoglobina A glicosilada y la disminución de peso durante el seguimiento de solo seis meses.

Finalmente, las metas del tratamiento para el control metabólico (glucemia, glucometría, hemoglobina A glicosilada y lípidos) deben individualizarse de acuerdo con edad, comorbilidades, complicaciones, expectativa de vida y red de apoyo.

\section{Conflictos de interés}

Ninguno.

\section{Financiación}

Ninguna.

\section{Bibliografía}

1. Kannel WB, McGee DL. Diabetes and cardiovascular disease The Framingham Study. JAMA. 1979;241:2035-8.

2. Einarson TR, Acs A, Ludwig C, Panton UH. Prevalence of cardiovascular disease in type 2 diabetes: a systematic literature review of scientific evidence from across the world in 2007-2017. Cardiovascular Diabetology. 2018;17(83.), 10.1186/s12933-018-0728-6.

3. Pinilla $A E$, Barrera MP, Sánchez AL, Mejía A. Factores de riesgo en diabetes mellitus y pie diabético: un enfoque hacia la prevención primaria. Rev Colomb Cardiol. 2013;20:213-22.

4. Pinilla AE, Barrera MP. Prevención en diabetes mellitus y riesgo cardiovascular: enfoque médico y nutricional. Rev Fac Med. 2018;63:459-518.

5. Cuenta de Alto Costo. Situación de la Hipertensión Arterial, Diabetes Mellitus y Enfermedad Renal Crónica 2015. Colombia. Disponible en: https://cuentadealtocosto. org/site/images/Situaci\%C3\%B3n_de_la_Enfermedad_Renal_ Cr\%C3\%B3nica_en_Colombia_2015.pdf.

6. Díaz J, Valdés $M$, Boullosa A. El trabajo interdisciplinario en la carrera de medicina: consideraciones teóricas y metodológicas. Medisur [Internet]. $2016 \mathrm{Abr}$ [Acceso 06 Nov 2018]; 14(2): 213-223.Disponible en: http://scielo.sld.cu/scielo.php?script=sci_arttext\&pid=S1727897X2016000200016\&lng=es.

7. Carvajal Y, Interdisciplinariedad:. desafío para la educación superior y la investigación. Revista Luna Azul; 2010;. 2018;31:156-69 [Acceso 6 Nov]. Disponible en: http://www.scielo.org.co/pdf/luaz/n31/n31a11.pdf.

8. American Diabetes Association. Standards of Medical Care in Diabetes; 2016. Diabetes Care. 2016; 39(Suppl. 1):S1-S112.

9. Asociación Latinoamericana de Diabetes. Guías ALAD sobre el Diagnóstico, Control y Tratamiento de la Diabetes Mellitus Tipo 2 con Medicina Basada en Evidencia.
Edición 2013. Rev ALAD. 2013;1-142. Disponible en: http://www.revistaalad.com/files/alad_v6_n4_181-192.pdf.

10. Ministerio de Salud y Proteccion Social. Guía de práctica clínica para el diagnóstico, tratamiento y seguimiento de la diabetes mellitus tipo 2 en la población mayor de 18 años. Guía No. GPC-2015-51. Sistema General de Seguridad Social en Colombia. Ministerio de Salud. Bogotá, 2016.

11. Canadian Diabetes Association Clinical Practice Guidelines Expert Committee. Clinical Practice Guidelines: Targets for Glycemic Control. Can J Diabetes. 2013:S31-4.

12. American Association of Clinical Endocrinologists and American College of Endocrinology. Consensus statement by the American Association of Clinical Endocrinologists and american college of Endocrinology on the comprehensive type 2 diabetes Management algorithm. Endocr Pract.2016;22:84-113.

13. International Diabetes Federation. Global Guideline por Type 2 Diabetes. 2012. [Acceso 29 nov 2016]. Disponibles en: http://www.idf.org/sites/default/files/IDF-Guideline-forType-2-Diabetes.

14. Friedewald W, Levy R, Fredrickson D. Estimation of the Concentration of Low-Density Lipoprotein Cholesterol in Plasma Without Use of the Preparative Ultracentrifuge. Clinical Chemistry. 1972;18:499-502.

15. McKee P, Castelli W, McNamara P, Kannel W. The natural history of congestive heart failure: the Framingham study. N Engl J Med. 1971;285:1441-6.

16. American College of Cardiology/American Heart Association Task Force on Clinical Practice Guidelines. AHA/ACC Guideline on the Management of Patients With Lower Extremity Peripheral Artery Disease. Circulation. 2016.

17. The International Council of Ophthalmology. ICO Guidelines for Diabetic Eye Care. 2016. [Citado 3 Mar 2017]. Disponible en: http://www.icoph.org/downloads/ICOGuidelinesforDiabetic EyeCare.pdf I.

18. International Society of Nephrology. Clinical Practice Guideline for the Evaluation and Management of Chronic Kidney Disease. Kidney Int Suppl. 2013;3:1-150.

19. IWGDF: Guidance on the management and prevention of foot problems in diabetes (internet). International Working Group on the Diabetic Foot. IWGDF guidance on the prevention of foot ulcers in at-risk patients with diabetes; 2015; [20 paginas]. Disponible en: http://iwgdf.org/guidelines/development-ofthe-iwgdf-guidance-documents-2015.

20. Barrera MP, Pinilla AE, Caicedo L, Castillo Y, Lozano Y, Rodríguez K. Factores de Riesgo Alimentarios y Nutricionales en adultos con Diabetes Mellitus. Rev Fac Med Univ Nac Colomb. 2012;60(1) Supl 1:1:28-40.

21. Alba L, Bastidas C, Vivas J, Gil F. Prevalencia de control glucémico y factores relacionados en pacientes con diabetes mellitus tipo 2 del Hospital Universitario de San Ignacio, Bogotá Colombia. Gac Méd Méx. 2009;145:469-74.

22. Jasso-Huamán LE, Villena A, Guevara-Linares X. Control metabólico en pacientes diabéticos ambulatorios de un hospital general. Rev Med Hered. 2015;26:167-72.

23. Khunti K, Wolden M, Larsen B, Andersen M, Davies M. Clinical Inertia in People With Type 2 Diabetes: A retrospective cohort study of more than 80,000 people. Diabetes Care. 2013;36:3411-7.

24. Calvert M, McManus R, Freemantle N. Management of type 2 diabetes with multiple oral hypoglycemic or insulin in primary care: retrospective cohort study. Br J Gen Pract. 2007;57: 455-60.

25. Lafata J, Dobie E, Divine G, Ulcickras M, McCarthy B. Sustained Hyperglycemia Among Patients with Diabetes: What matters when action is needed? Diabetes Care. 2009;32:1447-52.

26. Pesqueira P, Grandes J, Rodríguez C, Molinos S, González L, de La Cruz A, et al. Grado de control metabólico en pacientes 
ambulatorios con diabetes mellitus tipo 2 en Medicina Interna Estudio BARVI. Gac Médica Bilbao. 2012;109:52-8.

27. Pimouguet C, Le Goff M, Thiébaut R, Dartigues J, Helmer. Effectiveness of disease-management programs for improving diabetes care: a meta-analysis. CMAJ. 2011;183:E115-27.

28. Polinsky W, Earles J, Smith S, Pease D, Macmillan M, Christensen R, et al. Integrating Medical Management With Diabetes Self-Management Training: A randomized control trial of the Diabetes Outpatient Intensive Treatment program. Diabetes Care. 2003;26:3048-53.

29. Rotham R, Malone R, Bryant B, Shitani A, Crigler B, Darren $A$, et al. A randomized trial of a primary care-based disease management program to improve cardiovascular risk factors and glycated hemoglobin levels in patients with diabetes. Am J Med. 2005;118:276-84.

30. Osuna M, Rivera MC, Bocanegra CJ, Lancheros A, Tovar H, Hernández $\mathrm{Jl}$, et al. Caracterización de la diabetes mellitus tipo 2 y el control metabólico en el paciente hospitalizado. Acta Med Colomb 2014;39(4):344-51. Disponible en: http: / / www.scielo.org.co/scielo.php?script=sci_arttext\&pid=S0120$24482014000400007 \& \operatorname{lng}=e n$.

31. The DCCT research group. Diabetes Control and Complications Trial (DCCT): Results of Feasibility Study. Diabetes Care. 1987;10:1-19.

32. UK., Prospective Diabetes Study (UKPDS) Group. Intensive blood glucose control with sulphonylureas or insulin compared with conventional treatment and risk of complications in patients with type 2 diabetes (UKPDS 33). Lancet. 1998;352:837-53.

33. Turnbull F, Abrira C, Anderson R, Byington P, Chalmers J, Duckworth $\mathrm{W}$, et al. Intensive glucose control and macrovascular outcomes in type 2 diabetes. Diabetologia. 2009;52:2288-98.

34. Hemmingsen B, Lund S, Gluud C, Vaag A, Almdal T, Hemmingsen $C$, et al. Targeting intensive glycaemic control versus targeting conventional glycaemic control for type 2 diabetes mellitus. Cochrane Database of Systematic Reviews. 2011;15:1-247.

35. Eeg-Olofsson K, Cederholm J, Nilsson P, Zethelius B, Svensson A, Gudbjornsdòttir $\mathrm{S}$, et al. New aspects of HbA1c as a risk factor for cardiovascular diseases in type 2 diabetes: an observational study from the Swedish National Diabetes Register (NDR). J Intern Med. 2010;268:471-82.

36. Danaei G, Finucane M, Lu Y, Singh G, Cowan M, Paciorek C, et al. National, regional, and global trends in fasting plasma glucose and diabetes prevalence since 1980: Systematic analysis of health examination surveys and epidemiological studies with 370 country-years and 2,7 million participants. Lancet. 2011;378:31-40.

37. The National Cholesterol Education Program (NCEP). Guidelines for dyslipidemia treatment: adult treatment panel III (ATP-III). Endocrinol Nutr 2004; 51:254-65.
38. Gray N, Picone G, Sloan F, Yashkin A. The Relationship between BMI and Onset of Diabetes Mellitus and its Complications. South Med J. 2015;108:29-36.

39. Ashwell M, Gunn P, Gibson S. Waist-to-height ratio is a better screening tool than waist circumference and BMI for adult cardiometabolic risk factors: Systematic review and metaanalysis. Obes Rev. 2012;13:275-86.

40. Browning LM, Hsieh SD, Ashwell M. A systematic review of waist-to-height ratio as a screening tool for the prediction of cardiovascular disease and diabetes: 0.5 could be a suitable global boundary value. Nutr Res Rev. 2010;23:247-69.

41. De Koning L, Merchant AT, Pogue J, Anand SS. Waist circumference and waist-to-hip ratio as predictors of cardiovascular events: Meta-regression analysis of prospective studies. EurHeart J. 2007;28:850-6.

42. Czernichow S, Kengne AP, Stamatakis E, Hamer M, Batty GD. Body mass index, waist circumference and waist-hip ratio: which is the better discriminator of cardiovascular disease mortality risk?: evidence from an individual-participant metaanalysis of 82864 participants from nine cohort studies. Obes Rev. 2011;12:680-7.

43. Lim RB, Chen C, Naidoo N, Gay G, Tang WE, Seah D, et al. Anthropometrics indices of obesity, and all-cause and cardiovascular disease-related mortality, in an Asian cohort with type 2 diabetes mellitus. Diabetes Metab. 2015;41:291-300.

44. WHO. Obesity: Preventing and management the global epidemic. Report of a WHO Consultation on Obesity. World Health Organization. Geneva; 1997.

45. Gallagher D, Heymsfield S, Heo M, Jebb S, Murgatroyd P, Sakamoto $Y$. Healthy percentage body fat ranges: an approach for developing guidelines based on body mass index 1-3. Am J Clin Nutr. 2000;72:694-701.

46. Medina A, Ellis E, Ocampo D. Impacto de un programa personalizado de educación en pacientes diabéticos tipo 2. Acta Med Colomb. 2014;39:258-63.

47. Vyt A. Interprofessional and transdisciplinary teamwork. in health care. Diabetes Metab Res Rev 2008; 24(Suppl 1):S106-9.

48. Pinilla AE, Barrera MP, Rubio C, Devia D. Actividades de prevención y factores de riesgo en diabetes y pie diabético. Acta Med Colomb. 2014;39:250-7.

49. Pinilla AE. Enfoque terapéutico del paciente con diabetes mellitus 2. Nuevos antidiabéticos orales. En: Pinilla AE, Murgueitio R, Calderón CM, Mesa A, Villalobos W, Espinosa F, Vega M (editores). Compendio de Terapéutica en Medicina Interna. Evidencia actual. Sexta Edición. Bogotá: Editorial Celsus; 2016. p. 338-50.

50. Pinilla AE. Construcción y evaluación de un perfil de competencias profesionales en medicina interna. Colección Desarrollo Humano. Bogotá: Editorial Universidad Nacional de Colombia; 2015. 